\title{
Dust-ion-acoustic supersolitons in dusty plasmas with nonthermal electrons
}

\author{
Frank Verheest* \\ Sterrenkundig Observatorium, Universiteit Gent, Krijgslaan 281, B-9000 Gent, Belgium \& \\ School of Chemistry and Physics, University of KwaZulu-Natal, Durban 4000, South Africa \\ Manfred A. Hellberg ${ }^{\dagger}$ \\ School of Chemistry and Physics, University of KwaZulu-Natal, Durban 4000, South Africa \\ Ioannis Kourakis ${ }^{\ddagger}$ \\ Centre for Plasma Physics, Department of Physics and Astronomy, Queen's University Belfast, BT7 1NN Northern Ireland, United Kingdom
}

(Received 26 February 2013; published 15 April 2013)

\begin{abstract}
Supersolitons are a recent addition to the literature on large-amplitude solitary waves in multispecies plasmas. They are distinguished from the usual solitons by their associated electric field profiles which are inherently distinct from traditional bipolar structures. In this paper, dust-ion-acoustic modes in a dusty plasma with stationary negative dust, cold fluid protons, and nonthermal electrons are investigated through a Sagdeev pseudopotential approach to see where supersolitons fit between ranges of ordinary solitons and double layers, as supersolitons always have finite amplitudes. They therefore cannot be described by reductive perturbation treatments, which rely on a weak amplitude assumption. A systematic methodology and discussion is given to distinguish the existence domains in solitary wave speed and amplitude for the different solitons, supersolitons and double layers, in terms of compositional parameters for the plasma model under consideration.
\end{abstract}

DOI: 10.1103/PhysRevE.87.043107 PACS number(s): 52.27.Lw, 52.35.Fp, 52.35.Mw, 52.35.Sb

\section{INTRODUCTION}

Dusty plasmas are plasmas containing not only electrons, protons, and possibly other normal ions but specifically also charged dust grains. Prominent in typical heliospheric applications are micron-sized ice grains, which can pick up significant negative charge due to the much higher electron mobility. This leads to large charges and huge masses in comparison with the more usual ions [1,2]. In the early days of wave studies in dusty plasmas, Shukla and Silin [3] introduced the dust-ion-acoustic (DIA) mode, which is really an ion-acoustic wave modified by the imbalance between the undisturbed proton and electron densities.

On the scales related to the DIA mode, the dust dynamics can be neglected because of the large mass-per-charge compared to that of the protons. In the first instance, this leads to the simplest plasma model, where the negative dust is a stationary background, the protons are described by cold fluid equations, and the hot electrons are Boltzmann distributed [3]. Later, Bharuthram and Shukla [4] gave a fully nonlinear treatment of this DIA mode, with the help of Sagdeev pseudopotential theory [5].

Very recently, Dubinov and Kolotkov [6,7] introduced the novel concept of acoustic "super solitary waves" (or, with a colloquial, though less rigorous term, supersolitons) in a five-species dusty plasma but discussed only one single parameter case. Supersolitons can be characterized in various ways, either by looking at telltale wiggles (subsidiary maxima) on their electric field signature [6,7] or by requiring that the corresponding Sagdeev pseudopotential have three local ex-

\footnotetext{
*frank.verheest@ugent.be

†hellberg@ukzn.ac.za

¥i.kourakis@qub.ac.uk
}

trema between the undisturbed conditions and the supersoliton amplitude (thus yielding two contiguous "subwells") while remaining negative in this interval.

The modified bipolar electric field structure that is characteristic of a supersoliton is a signature that should be found in space-based observations, and, indeed, there are indications that it may well have been recorded [8]. Since the description of solitary waves is usually given in terms of the electrostatic potential, not of the associated electric field, pseudopotentials with three local extrema were encountered earlier [9-12] but not recognized as differing from ordinary solitons.

In particular, it was pointed out by Verheest et al. [13] that several three-species models could have supersoliton solutions and, hence, that their importance warranted a more systematic approach to their characteristics and existence domains in parameter space. Hence, in the present paper we investigate a DIA model, comprised of infinitely massive negative dust and cold positive ions, together with nonthermal Cairns distributed [14] electrons. It will be recalled that Cairns et al. [14] introduced this velocity distribution function to model the excess of superthermal particles, often observed in space plasmas, as a vehicle to explore their effects on nonlinear waves in space.

Many studies of nonlinear DIA waves have been based on small-amplitude methods such as a Korteweg-de Vries (KdV) reductive perturbation treatment. It should be emphasized that supersoliton profiles cannot be described by such an approach. Hence, from the extensive literature on nonlinear DIA waves we shall only quote a selection of the relevant papers which deal with large-amplitude modes through the arbitrary amplitude Sagdeev pseudopotential analysis or equivalent fluid dynamical approach $[15,16]$. These include Refs. [17-23]. Baluku et al. [24] investigated DIA solitons in a plasma composed of mobile dust, warm ions, and $\kappa$-distributed [25-27] electrons, including the limit $\kappa \rightarrow \infty$, 
which represents a Maxwellian distribution. In addition to exploring existence domains in parameter space and effects of ion temperature, they evaluated the soliton amplitudes systematically as various parameters were varied, as well as considering effects of finite dust inertia.

The paper is structured as follows. In the following section we set out the basic formalism, obtain the Sagdeev pseudopotential, and outline the process of analysis. Section III is devoted to a thorough numerical study of two typical cases and a discussion of the limitations of the model. This is followed by a short summary of the results.

\section{BASIC FORMALISM}

The theoretical model includes immobile negative dust, cold positive ions, and nonthermal electrons, labeled $d, i$, and $e$, respectively. The fraction of negative charge residing on the dust is $f=z_{d} n_{d 0} / z_{i} n_{i 0}$ and on the electrons $1-f=$ $n_{e 0} / z_{i} n_{i 0}$, where $n_{i 0}$ is the undisturbed ion density. The number of charges on the ions and the dust are $z_{i}$ and $z_{d}$, respectively.

The ions are described by the continuity and momentum equations in normalized variables [28], referred, among others, to a speed $C_{a}=\left(z_{i} T_{e} / m_{i}\right)^{1 / 2}$, where $T_{e}$ is the kinetic temperature of the electrons in the absence of nonthermal effects. The present normalization implies that the space coordinate is measured in units of $\left(\varepsilon_{0} T_{e} / n_{i 0} z_{i} e^{2}\right)^{1 / 2}$. In a frame where the nonlinear structure is stationary $(\partial / \partial t=0)$, all variables tend to their undisturbed values at $x \rightarrow-\infty$, and, in particular, the electrostatic potential $\varphi$ (normalized to $T_{e} / e$ ) tends to zero. All densities will be normalized with respect to their equilibrium values.

One can integrate the ion equations with respect to $x$ and find that the ion charge density is given by

$$
n_{i}=\frac{1}{\sqrt{1-\frac{2 \varphi}{M^{2}}}} .
$$

We note that the ion density contains $M=V / C_{a}$, coming from inertial effects on their flow, where $V$ is the as-yet-unknown velocity of the nonlinear structure, as seen in an inertial frame. There are limitations on $\varphi$ on the positive side at $\varphi_{\ell i}=M^{2} / 2$, as for larger $\varphi$ the ion density is no longer defined and, in fact, reaches infinite compression for $\varphi \rightarrow \varphi_{\ell i}$.

As noted in the Introduction, we will adopt a nonthermal Cairns distribution function for the electrons. The phasespace Cairns distribution function is expressed in terms of a nonthermality parameter $\alpha$ [14] and yields, after integration, the electron charge density as [28]

$$
n_{e}=\left(1-\beta \varphi+\beta \varphi^{2}\right) \exp (\varphi) .
$$

Here the macroscopic nonthermality parameter is defined as $\beta=4 \alpha /(1+3 \alpha)$. In the discussion below, values of $\beta$ smaller than $4 / 7$ (corresponding to $\alpha=0.25$ ) will be used, since at larger $\alpha$ or $\beta$ the phase-space Cairns distribution function develops strong wings and this nonmonotonic form may lead to linear instability, thus precluding the generation of stable nonlinear structures [28]. This then renders it less appropriate to model superthermal particles, for which more sophisticated descriptions are needed.
The basic set of equations is closed by Poisson's equation,

$$
\frac{d^{2} \varphi}{d x^{2}}+n_{i}-(1-f) n_{e}-f=0
$$

where the term $f$ corresponds to the stationary negative dust contribution, as defined above, and we have used overall charge neutrality in the undisturbed conditions. After integration, (3) yields an energy-like integral,

$$
\frac{1}{2}\left(\frac{d \varphi}{d x}\right)^{2}+S(\varphi, M)=0,
$$

which can be analyzed as in classical mechanics, in terms of a Sagdeev pseudopotential [5]

$$
\begin{aligned}
S(\varphi, M)= & (1-f)\left[1+3 \beta-\left(1+3 \beta-3 \beta \varphi+\beta \varphi^{2}\right) \exp (\varphi)\right] \\
& -f \varphi+M^{2}\left(1-\sqrt{1-\frac{2 \varphi}{M^{2}}}\right) .
\end{aligned}
$$

Here we have explicitly referred to $M$, while parameters like $f$ and $\beta$ determine the precise composition of the plasma.

By construction and assumption $S(0, M)=S^{\prime}(0, M)=0$ and $S^{\prime \prime}(0, M) \leqslant 0$ is required to cause the origin to be a (local) unstable maximum, at least on one side. Derivatives of $S(\varphi, M)$ with respect to $\varphi$ are denoted by primes. In physical terms, the proper convexity condition, $S^{\prime \prime}(0, M) \leqslant 0$, ensures that the nonlinear structures are (super-)acoustic in a global sense and this yields the minimal $M$ for their existence,

$$
M^{2} \geqslant M_{s}^{2}=\frac{1}{(1-f)(1-\beta)} .
$$

Here $M_{s}$, which satisfies $S^{\prime \prime}\left(0, M_{s}\right)=0$, is the true normalized acoustic speed in the plasma system. Hence, the ratio $M / M_{s}$ is the true Mach number in the system, since the reference speed used in the normalization disappears from this ratio.

However, in order to have a solitary wave solution, one needs to encounter a root of $S(\varphi, M)$ outside and accessible from $\varphi=0$. Single roots give hill- or diplike solitary waves, whereas for double roots $\varphi$ changes from one value at $-\infty$ to another at $+\infty$, typical for potential kinks.

We note that $S(\varphi, M) \rightarrow+\infty$ for $\varphi \rightarrow-\infty$, due to the dominance of the dust contribution for large negative $\varphi$. Hence, there is always at least one negative root, and possibly more, but the number of negative roots will be shown always to be odd. As will be seen later, very large values of $|\varphi|$ are not admissible, since then some of the assumptions underlying the model break down. There are two crucial assumptions, the neglect of dust dynamics and of electron inertia, which will be discussed in Sec. III C.

In addition to the negative $\operatorname{root}(\mathrm{s})$, there might also be positive roots, but their existence cannot be predicted by reasoning on general properties of $S(\varphi, M)$ alone, only that any possible positive roots need to satisfy $\varphi<\varphi_{\ell i}$, for the pseudopotential to be defined.

In the following sections the existence domains for nonlinear solitary waves and kinks are analyzed in a systematic way, rather than considering only a limited set of $M$ values and compositional parameters, for which the numerics give solutions.

We also compute

$$
S^{\prime \prime \prime}\left(0, M_{s}\right)=(1-f)\left[3(1-f)(1-\beta)^{2}-1\right] .
$$


The sign of $S^{\prime \prime \prime}\left(0, M_{s}\right)$, given by the sign of the expression between the square brackets in (7), determines the sign of the KdV-like solitons [24,29]. By "KdV-like" we mean that such solitons have amplitudes which become arbitrarily small as $M \rightarrow M_{s}$, as do solutions of KdV equations [24]. Because generically $\partial S / \partial M<0$ [30], for a specified plasma composition, the family of curves $S(\varphi, M)$ all touch at $\varphi=0$ but otherwise cannot cross, as $M$ is varied. This implies that soliton roots of $S(\varphi, M)$ (the first accessible roots from $\varphi=0$ on the positive and/or negative side) increase in amplitude as $M$ is increased. If $M / M_{s}$ can be increased significantly, so can the soliton amplitudes, and, thus, KdV-like solitons are not always small, contrary to what happens in weakly nonlinear descriptions [1].

The Sagdeev pseudopotential at the acoustic speed, $S\left(\varphi, M_{s}\right)$, has other interesting properties. Bearing in mind that $S^{\prime \prime}\left(0, M_{s}\right)=0$ by definition, we see that for $S^{\prime \prime \prime}\left(0, M_{s}\right)<0$, $S\left(\varphi, M_{s}\right)$ goes from positive to negative values as one increases $\varphi$ through zero. Thus, the pseudopotential cannot have a negative soliton root but might have an acceptable root for positive $\varphi$. If this is indeed the case, this positive soliton is additional to the negative KdV-like solitons and at the same time constitutes a minimum for possible other solitons for increasing $M$, which we therefore have called "non-KdVlike" $[10,24,29]$. For $S^{\prime \prime \prime}\left(0, M_{s}\right)>0$ the whole reasoning is negative/positive inverted. Because of their finite amplitudes at $M=M_{s}$, these non-KdV-like solutions cannot be obtained through a weakly nonlinear, reductive perturbation approach.

Whenever there is a possibility that for certain parameter values $S^{\prime \prime \prime}\left(0, M_{s}\right)$ goes from one sign to the opposite sign, the soliton polarities and characteristics will switch accordingly. Clearly, the plasma composition parameter values that give rise to the switch are critical $[10,24,29]$, and in this model one is led to critical values of the nonthermality parameter, $\beta_{c}$, and dust charge fraction, denoted by $f_{c}$. It is possible with this technique to find numerous realistic plasma models in which, for some fixed plasma parameter values, both polarities are supported [4,10,14,17,24,28,31,32], a phenomenon sometimes referred to as "coexistence" [24,28-30,32-34]. Even though positive and negative solitons can occur for the same parameter values in the Sagdeev pseudopotential, only one possibility can be realized at a time, depending on the initial perturbations. This is analogous to the situation in classical mechanics, where a sphere, placed on top of a local hill, can, in principle, roll down on either side but not on both sides simultaneously.

\section{DISCUSSION}

To get a feeling for where interesting phenomena might occur, we start the discussion by plotting in Fig. 1 how the critical density fraction, $f_{c}$, varies with $\beta$ along the curve $S^{\prime \prime \prime}\left(0, M_{s}\right)=0$. It is seen from Fig. 1 that, above this curve, $S^{\prime \prime \prime}\left(0, M_{s}\right)<0$, which yields negative potential KdV-like solitons. On the other hand, below $S^{\prime \prime \prime}\left(0, M_{s}\right)=0$, $\mathrm{KdV}$-like solitons are positive. From (7) one also notes that for $\beta \geqslant \beta_{c}=1-1 / \sqrt{3} \simeq 0.423$ only $S^{\prime \prime \prime}\left(0, M_{s}\right)<0$ is possible, hence, no polarity changes can occur and the KdV-like solitons are negative for all $f$. We will thus pick two specific illustrative cases, one in each of these two regions.

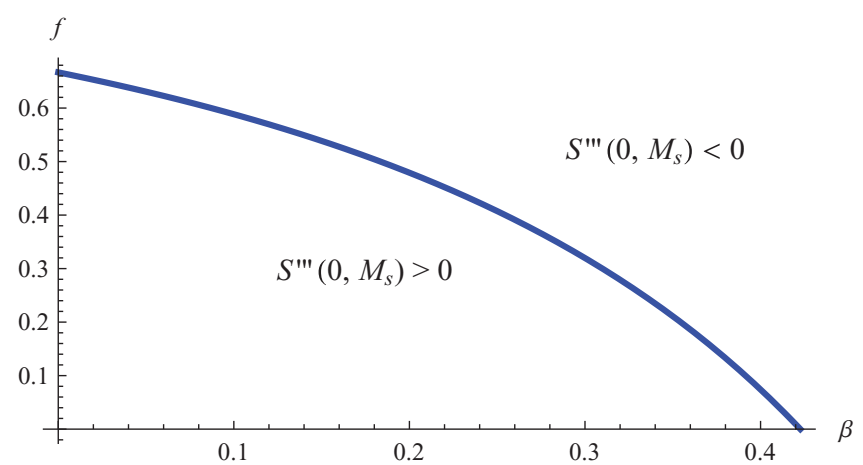

FIG. 1. (Color online) Variation of the critical charge density fraction, $f_{c}$, with $\beta$, along the curve $S^{\prime \prime \prime}\left(0, M_{s}\right)=0$, across which the polarity changes. Above this curve, $S^{\prime \prime \prime}\left(0, M_{s}\right)<0(\mathrm{KdV}$-like solitons are negative), while below $S^{\prime \prime \prime}\left(0, M_{s}\right)>0$ (with positive $\mathrm{KdV}$-like solitons).

One is $\beta=0.5$ (corresponding to $\alpha=0.2$ ), a standard value in many discussions using the Cairns nonthermal distribution (for example, Refs. [14,21,30,32,35-39]), clearly lying in the region where a polarity switch does not take place. As a second example we choose $\beta=0.3$, in the domain where the $\mathrm{KdV}$ and non-KdV-like polarities will switch at $f=0.320$, as $f$ is increased. Both values of $\beta$ are chosen to lie in the physically important range $0 \leqslant \beta<4 / 7$, because, as pointed out above, we prefer not to consider larger values [28]. The discussion of the case $\beta=0.3$ being more involved, we will therefore begin with it.

Supersolitons require pseudopotentials with three local extrema or two distinct wells between $\varphi=0$ and a negative or a positive root. It follows immediately from (4) that each extremum in $S(\varphi, M)$ must be reflected in a positive and a negative extremum in the electric field, thereby generating the characteristic signature of a supersoliton. We briefly recall that limits on the existence ranges for supersolitons involve first double layers $[10,13]$, which, if they exist, are always lower limits, whereas coalescence of two of the three local extrema, thereby merging the two pseudopotential wells, can act as lower or upper limits. Further details are given in Ref. [13]. These limitations can be drawn as curves in $\left\{f, M / M_{s}\right\}$ or in $\{f, \varphi\}$ parameter space, showing the changes as $f$ is increased. All this will be illustrated in more detail below.

\section{A. Case with $\beta=0.3$}

We now address a case where $\beta=0.3<\beta_{c}$, so polarity changes are possible for the KdV-like solitons and, if they exist, for the non-KdV-like solitons, and these changes occur at the critical value $f_{c}=0.320$. To begin the discussion, we note that in the range $0<f<0.320$ we have $S^{\prime \prime \prime}\left(0, M_{s}\right)>0$, so there are positive $\mathrm{KdV}$-like solitons. As pointed out in Sec. II, this model always has at least one negative root, and the associated negative solitons are, thus, clearly non-KdV-like. For $0.320<f$, there are negative $\mathrm{KdV}$-like solitons, and over a limited range, positive non-KdV-like solitons. However, such interchanges in the polarity and character of the different soliton modes do not give rise to discontinuous changes.

To refine the discussion, we consider the behavior as $f$ is increased from zero and illustrate this with two figures, one 


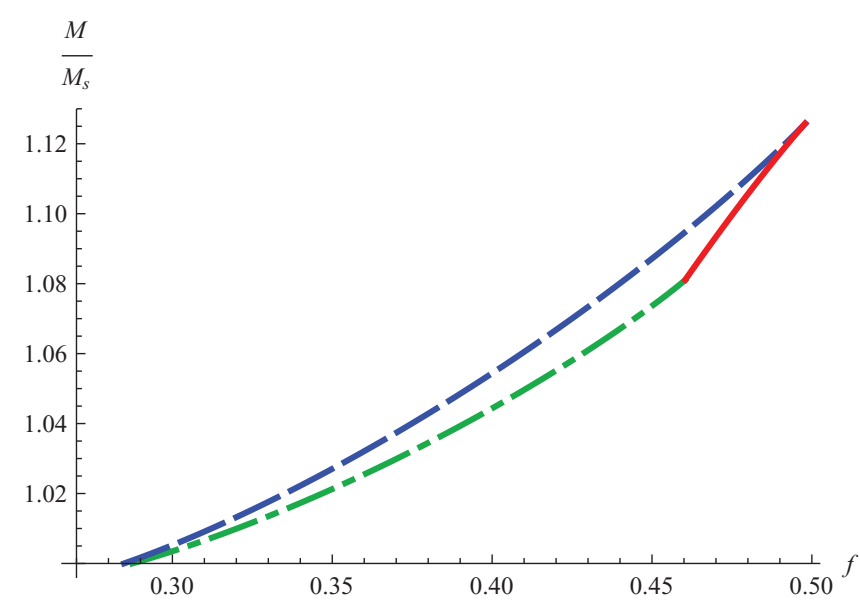

FIG. 2. (Color online) For $\beta=0.3$, the region where negative supersolitons can be found is above the green dotted-dashed curve (occurrence of negative double layers) or the full red curve $(A B$ coalescence) and below the blue dashed curve ( $B C$ coalescence). Note that both the dashed and dotted-dashed curves start at the acoustic speed, $M=M_{s}$, but at slightly different $f$.

for the Mach number ranges (in Fig. 2, restricted for clarity to the supersoliton domain) and another for the amplitudes of ordinary solitons, double layers, and supersolitons in Fig. 3. In this way it will become clear how the figures have been constructed.

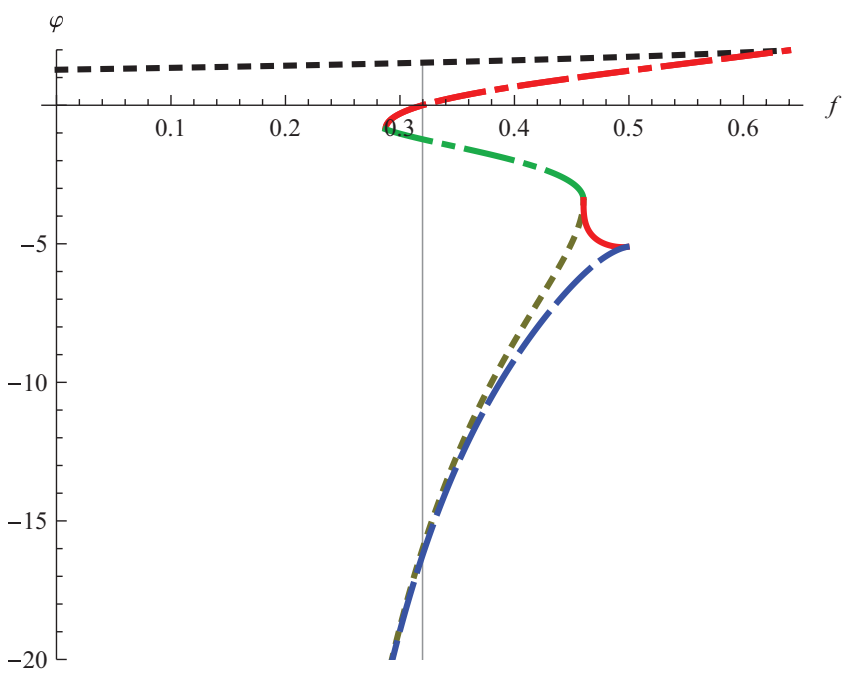

FIG. 3. (Color online) Existence ranges for positive and negative solitons and supersolitons, given for $\beta=0.3$ in terms of the amplitudes. The black upper dotted curve represents a cutoff due to infinite ion compression, whereas the red dotted-double-dashed curve denotes acceptable roots at the acoustic speed. The negative acoustic roots that occur for low values of $f$ have been omitted for reasons of graphical clarity, as they have very large amplitudes. The thin gray line at $f_{c}=0.320$ indicates where the polarity changes. Further, the green dotted-dashed curve indicates negative double layers, the red solid curve where an $A B$ coalescence occur, the blue dashed curve refers to a $B C$ coalescence, and the dark gray lower dotted curve is the minimum supersoliton amplitude.
In the first range, for $0<f<0.287$, i.e., within the range of positive potential $\mathrm{KdV}$ solitons, one can check that there is a (very large) negative non-KdV-like root at the acoustic speed, $M_{s}$, of amplitude $\left|\varphi_{a}\right|>21$. We have seen that $S(\varphi, M)$ always admits at least one negative root, and the negative non-KdV-like solitons that are found for $M>M_{s}$ have even larger amplitudes, without apparent limit as $M$ is further increased. However, the very large modes in this range have been omitted from the comprehensive graph in Fig. 3 for reasons of graphical clarity. We note that for $0<f<0.285$ there are no supersolitons.

At $f=0.287$ a negative double layer appears at the acoustic speed $M_{s}$, with amplitude $\varphi=-0.865$, in addition to a large inaccessible, physically unimportant, negative root at $\varphi=$ -20.976. To compute the double-layer amplitudes and Mach number, we have to solve numerically the set $S(\varphi, M)=0$ and $S^{\prime}(\varphi, M)=0$, at given compositional parameters, and repeat the process for, for example, values of $f$ in a range.

From $f=0.287$ onwards pseudopotentials with $M_{s}<M<M_{\mathrm{dl}}$ have three negative roots, of which only the smallest in amplitude is accessible from the undisturbed conditions, thus representing a soliton amplitude. As $f$ is increased, the gap between these three roots decreases, until for $f=0.460$ they all coalesce in a triple root. The picture for $0.287<f<0.460$ is that, at given $f$ and increasing $M$, one encounters an accessible negative root for $M_{s}<M<M_{\mathrm{dl}}$ yielding a normal negative soliton, until a double layer is obtained at $M=M_{\mathrm{dl}}$. For larger $M>M_{\mathrm{dl}}$, the domain beyond the third root of $S\left(\varphi, M_{s}\right)$ suddenly become accessible, and this is where one enters the supersoliton regime for given $f$ in the range $0.287<f<0.460$.

It is worth stressing that the increase in $M$ above $M_{\mathrm{dl}}$ occurs continuously, but the amplitudes jump in a discontinuous way $[10,13]$ from the double-layer amplitude to beyond the third root of $S\left(\varphi, M_{S}\right)$, a gap which can be quite appreciable, as we will see in a detailed example below. This phenomenon was first reported in Ref. [10], before the introduction of the nomenclature supersoliton. Hence, the third root of the double-layer pseudopotential represents a minimum for the supersoliton amplitude at the same plasma composition.

Further increases in $M$ will cause a coalescence of two of the local extrema within the pseudopotential, merging the two pseudopotential subwells on the negative side and signaling the end of the supersoliton range. Beyond the value of $M$ at which this coalescence occurs, we recover standard solitons, and this transition is continuous, both in $M$ and in amplitude.

In between, at $f=0.320$, we have crossed the value where the characteristics of the negative- and positive-polarity solitons are swapped from $\mathrm{KdV}$ - to non-KdV-like or vice versa. As we see in Figs. 2 and 3, this transition is continuous both in $M$ and in amplitude, as, in particular, the KdV-like solitons are not necessarily small for all $M$ but start from $M>M_{s}$ and increase proportionally to $M-M_{s}$. This is also illustrated in Fig. 4, which clearly shows that, as $f$ is increased, the gap between the negative roots becomes smaller, whereas the positive soliton amplitude increases.

For $f>0.460$, there are no more double layers and the pseudopotentials only have one negative root. However, as discussed in our earlier paper [13], supersolitons can still be found for larger $f>0.460$, beyond the existence range of 

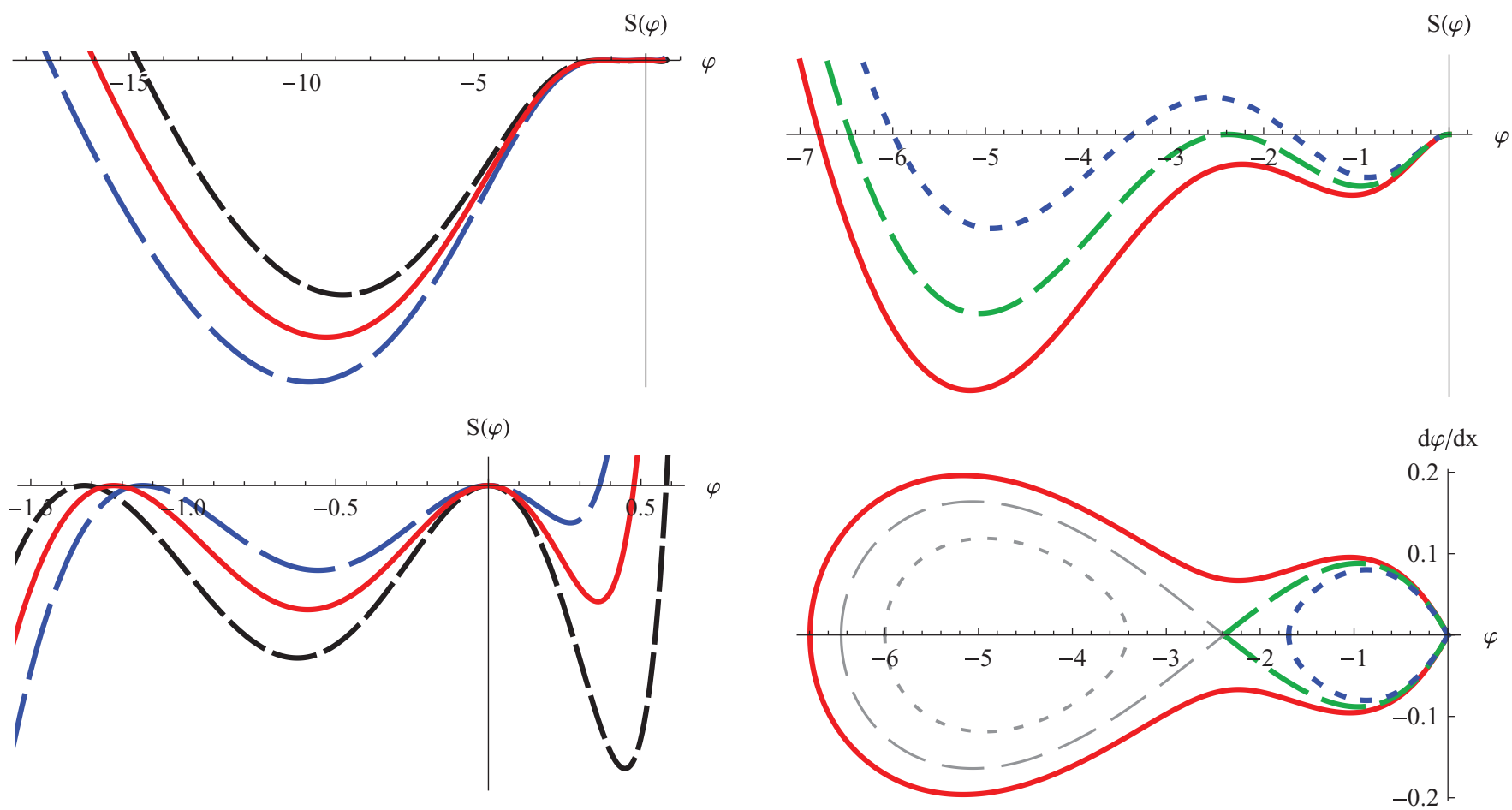

FIG. 4. (Color online) Upper panel: Pseudopotentials with a negative double layer for $f=0.31$ (blue curve with longer dashes), $f=0.320$ (red solid curve, at the precise polarity crossover), and $f=0.33$ (black curve with shorter dashes). Lower panel: Focus on the $\varphi$ range closer to the undisturbed conditions. Both panels together show that as $f$ is increased, the absolute values of the large negative root decrease, whereas the amplitudes of the negative double layers and the positive soliton increase.

double layers. For a given value of $f$, the lower limit in $M$ of the supersoliton existence domain is then governed by the emergence of two subwells within the main pseudopotential well. Associated with this, two new extrema are added in the well. As $M$ is increased further, one finds a coalescence between two of the local extrema, thereby merging the two subwells and providing an upper limit for supersolitons, as is the case when double layers form the lower limit.

To understand the nomenclature that we use in Ref. [13], we have labeled the three local extrema $A, B$, and $C$, going from left to right [i.e., from the root of $S(\varphi, M)$ ] in the direction of $\varphi=0$, as we shall also use in Fig. 9 below. Two types of coalescence are possible: the two leftmost $(A B)$ or the two rightmost $(B C)$ extrema. We have noted in our earlier study [13] that the former coalescence always occurs at lower speed than the latter, i.e., $M_{A B}<M_{B C}$, and that, as long as double layers occur, an $A B$ coalescence is not possible. Beyond the double-layer range in $f$, supersolitons can be found in the $M$ range between these two coalescences. This will also be confirmed here, as seen in Fig. 5, when an explicit example is discussed. The end of the supersoliton range is reached when the three local extrema coalesce, in an $A B C$ coalescence, that is, the lower and upper limits coincide. For $\beta=0.3$ this occurs on the negative potential side at $f=0.498$.

For $f>0.498$, only $S(\varphi, M)$ curves with one negative root are possible. These correspond to a standard soliton, the amplitude of which increases with $M$. Again, these can be

FIG. 5. (Color online) Upper panel: Pseudopotentials with a standard soliton (blue dotted curve, $M / M_{s}=1.057$ ), a double layer (green dashed curve, $M / M_{s}=1.061$ ), and a supersoliton (red solid curve, $M / M_{s}=1.065$ ) for $\beta=0.3$ and $f=0.43$. For graphical clarity, the positive soliton domain has been omitted, because the well on this side is very deep and would flatten the important details on the negative side. Lower panel: Here the hodographs are presented, plotting $d \varphi / d x$ as functions of $\varphi$, with the same curve coding. Thin dotted and dashed curves in gray indicate ranges which are not accessible from the undisturbed conditions.

arbitrarily large, as there is no explicit upper limit in the model, but we will have to qualify that statement in Sec. III C.

Turning now to the standard positive solitons, the existence of which is limited by $\varphi_{\ell i}$, defined in Sec. II, we note from Fig. 3 that for $f<0.320$ the positive solitons are KdV-like, and that their limiting amplitude $\varphi_{\ell i}$ increases with $f$. Once we cross over to $f>0.320$, the positive solitons become non$\mathrm{KdV}$-like, and now their minimum amplitude increases faster than the maximum at $\varphi_{\ell i}$, so this range ends for $f=0.639$, when the root at the acoustic speed occurs at the corresponding $\varphi_{\ell i}=M_{s}^{2} / 2$. Since there is always at least one negative soliton, the coexistence domain that supports both polarities of solitons covers $0<f<0.639$.

In the existence diagrams, Figs. 2 and 3, it can be seen that the domain for the supersolitons forms a wedge, both in Mach number $M / M_{S}$ space and in amplitude space. The corners in Fig. 2, and similarly in Fig. 3, can be determined precisely at $f=0.285$ ( $B C$ coalescence at the acoustic speed), $f=0.287$ (double layers at the acoustic speed), $f=0.460$ (triple root signaling the end of the double-layer range), and $f=0.498$ (coalescence of all local extrema in the well). Note that we have chosen to plot $M / M_{s}$, as that does away with the choice of velocity normalization and gives the true Mach number of the solitons. We observe that this shape is similar to that found 

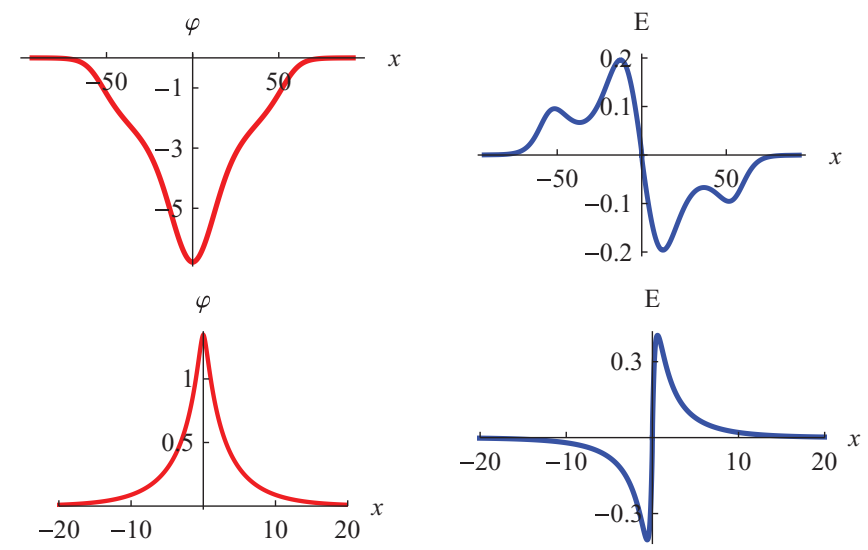

FIG. 6. (Color online) Upper panel: Profile (left) and electric field (right) of the negative supersoliton belonging to the pseudopotential shown by the solid curve in Fig. 5. Lower panel: Profile (left) and electric field (right) of the standard positive soliton belonging to the same pseudopotential, which was omitted in Fig. 5 for reasons of graphical clarity, the well on the positive side being very deep.

previously for a plasma composed of Cairns electrons and cold positive and negative ions [13]. The tiny supersoliton regime between $f=0.285$ and 0.287 can hardly be seen in Figs. 2 and 3 . It is at the limit of numerical precision and presumably too small to be of physical significance.

Having determined the existence domains for the Mach numbers in Fig. 2 and for the amplitudes in Fig. 3, we can draw as many Sagdeev pseudopotentials as we want but will only select a few values of $f$ to illustrate in somewhat more detail what was sketched above. We start with a dust charge density fraction $f=0.43$, which leads to Figs. 5 and 6 .

In the upper panel of Fig. 5 we have plotted three pseudopotentials, associated with a standard soliton (dotted curve, $M / M_{s}=1.057$ ), a double layer (dashed curve, $M / M_{s}=$ 1.061 ), and a supersoliton (solid curve, $M / M_{s}=1.065$ ), respectively. For reasons of graphical clarity, the range $\varphi>0$ has been omitted, but all three pseudopotentials have a normal, positive soliton of amplitudes 1.311, 1.331, and 1.348, respectively.

With the same curve coding the lower panel of Fig. 5 gives the corresponding hodographs, plotting $d \varphi / d x(=-E)$ as a function of $\varphi$. Similar hodographs have been found previously in other plasma configurations $[6,10,13]$. Here the jump from the double layer to the supersoliton amplitude is clearly seen, even though the Mach numbers vary continuously. Thin dotted and dashed curves in gray indicate parts of the hodograph that are not accessible from the undisturbed conditions at $\varphi=0$, yielding another characteristic of supersolitons [6,13].

Figure 6 gives, for the supersoliton pseudopotential depicted in Fig. 5 by the solid curve, both soliton and electric field profiles for the negative supersoliton (upper panel) and the standard positive soliton (lower panel). The wiggles superposed on a bipolar electric field structure are clearly seen, whereas the soliton plots themselves show a much less obvious difference. These figures are reminiscent of previous observations in other plasmas $[6,7,13]$.

Next, we look at the graphs corresponding to $f=0.460$ where the three negative roots merge into a triple root, Figs. 7

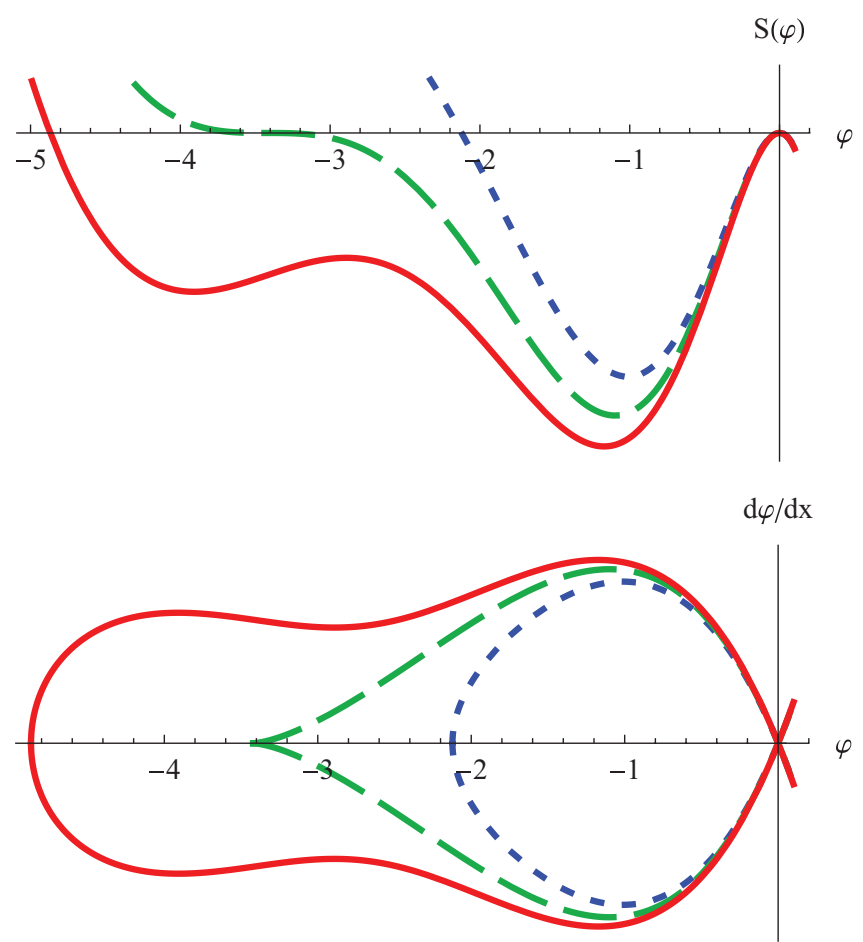

FIG. 7. (Color online) Upper panel: Pseudopotentials with a standard soliton (blue dotted curve, $M / M_{s}=1.077$ ), a triple root (green dashed curve, $M / M_{s}=1.081$ ), and a supersoliton (red solid curve, $M / M_{s}=1.084$ ) solution for $\beta=0.3$ and $f=0.460$. For graphical clarity, the positive range has been omitted. Lower panel: Corresponding hodographs, plotting $d \varphi / d x$ as functions of $\varphi$.

and 8. The pseudopotential represented in Fig. 7 by a dashed curve has a triple root, whereas those for a smaller or larger $M$ only have one root in the negative domain. The smaller root (on the dotted curve) signals a standard soliton, the larger root (on the solid curve) a supersoliton. However, when $M$ is increased too much, a $B C$ coalescence occurs and the supersoliton range ends. Still higher values of $M$ correspond again to normal solitons.

In Fig. 8 the supersoliton profile and electric field for the pseudopotential with the solid curve in Fig. 7 show a marked difference from the profiles given in the upper panel of Fig. 6. The position of the highest of the peaks in the modified electric field corresponds to the deepest well in the corresponding pseudopotential, as one may deduce immediately from (4). Note that the undisturbed conditions at $\varphi=0$ are for $|x| \rightarrow \infty$, whereas $E=0$ or $d \varphi / d x=0$ occur at the peak of the soliton,
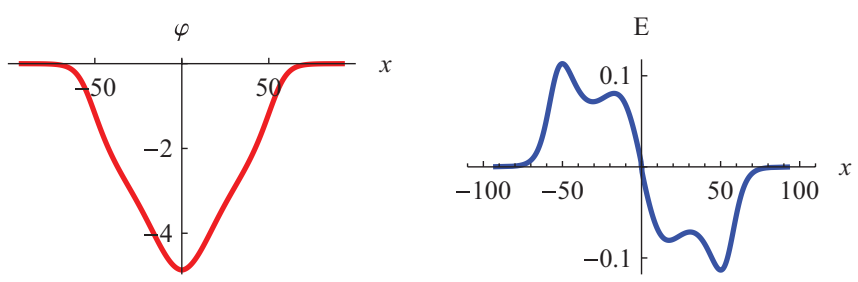

FIG. 8. (Color online) Profile (left) and electric field (right) of the negative supersoliton belonging to the pseudopotential shown by the solid curve in Fig. 7. 

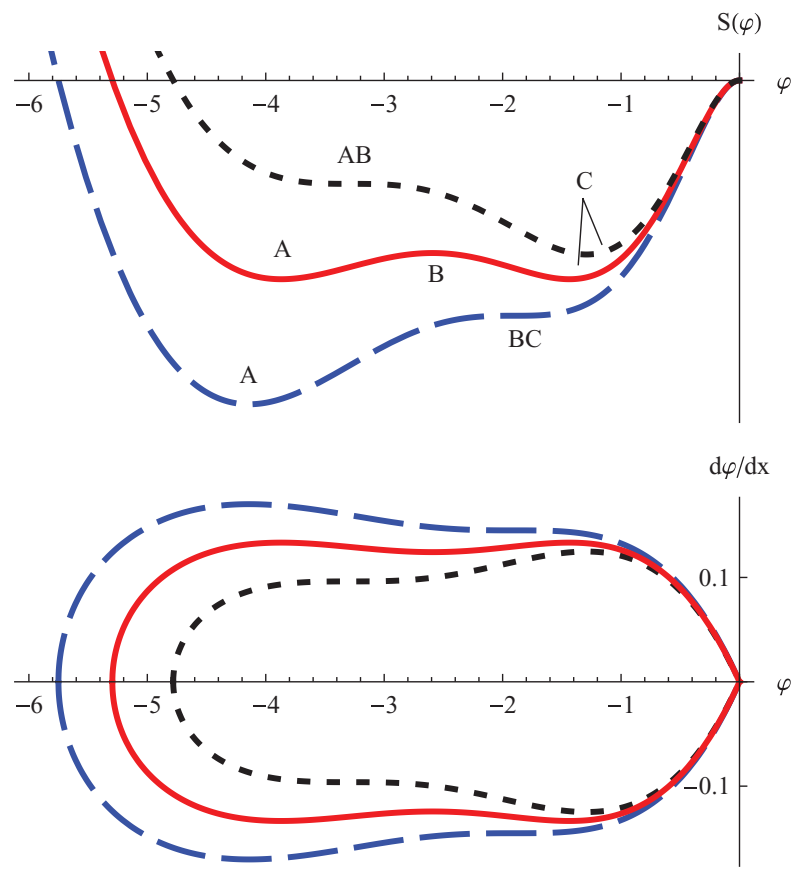

FIG. 9. (Color online) Upper panel: Examples of pseudopotentials yielding a supersoliton (red solid curve, with local extrema $A, B$, and $C$, for $M / M_{s}=1.097$ ), between two standard solitons [coalescence of two local extrema, $A$ and $B$ (black dotted curve, for $M / M_{s}=1.093$ ) or $B$ and $C$ (blue dashed curve, for $M / M_{s}=1.102$ ), respectively], at $\beta=0.3$ and $f=0.47$. For graphical clarity, the positive soliton domain has been omitted. Lower panel: Corresponding hodographs, plotting $d \varphi / d x$ as functions of $\varphi$.

at $x=0$. Comparing the wells for the solid curves in Figs. 5 and 7 corroborates this.

To conclude this subsection, we give in Figs. 9 and 10 an example of supersolitons at $f=0.47$, in the range where all pseudopotentials have only one negative root. Here the pseudopotential yielding a supersoliton (solid curve, with local extrema $A, B$, and $C$, for $M / M_{s}=1.097$ ) is found between two pseudopotentials with standard solitons. There is an $A B$ coalescence of two local extrema (dotted curve, for $M / M_{s}=$ 1.093) or a $B C$ coalescence (dashed curve, for $M / M_{s}=$ 1.102 ), such that $M_{A B}<M_{B C}$, as also found previously [13]. We note that if one is considering increasing $M$, what is termed the $A B$ coalescence here manifests itself as the emergence of the two subwells within the pseudopotential well, as two new local extrema are generated. To compute the Mach
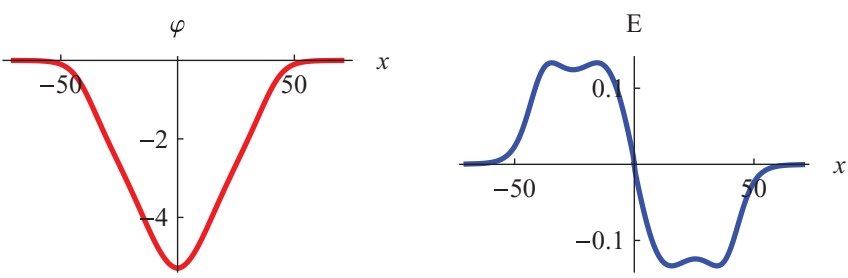

FIG. 10. (Color online) Profile (left) and electric field (right) of the negative supersoliton belonging to the pseudopotential shown by the solid curve in Fig. 9 . numbers at which an $A B$ or $B C$ coalescence occurs, we have to solve numerically the set $S^{\prime}(\varphi, M)=0$ and $S^{\prime \prime}(\varphi, M)=0$ for $M$ and then find the root of the pseudopotential for that $M$ at given compositional parameters.

As the electric field signature also shows, this is an example (carefully chosen) of a pseudopotential where the two wells of the supersoliton pseudopotential are equally deep, resulting in equal local maxima or minima in the electric field, a property hardly discernible on the supersoliton electrostatic potential profile itself.

\section{B. Case with $\beta=0.5$}

For the case where $\beta=0.5$, the reasoning follows lines analogous to the previous subsection, with the major difference that there is no polarity change, so the negative solitons are $\mathrm{KdV}$-like throughout the range and the positive solitons non$\mathrm{KdV}$-like. In this case, the positive range ends at $f=0.350$ due to infinite ion compression, at $\varphi_{\ell i}$, up to which value of charge density fraction there is coexistence with the negative solitons.

In an analogous way to that by which Fig. 2 was established, we find in Fig. 11 the Mach number range which allows supersolitons to occur. We see again a wedge shape, but, interestingly, the lower cutoff in soliton speed is not at the acoustic speed and finite $f$ but occurs at $f=0$ and $M>M_{s}$. As the negative roots are KdV-like in this case, standard (negative) solitons start at very small amplitudes for $M \simeq M_{s}+\delta$. The first limit on solitons is given by a double layer and, thus, occurs at $M_{\mathrm{dl}} / M_{s}>1$, and any supersolitons only arise for $M>M_{\mathrm{dl}}>M_{s}$.

A second major difference with the case of $\beta=0.3$ is that even for small $f$, double layers can occur and, hence, for $M>M_{\mathrm{dl}}$, supersolitons can also occur, the existence domain of which now spans a wide range in $f$. However, while in theory this could already start at $f=0$, this value is not admissible in the model considered, as then there is no dust present. The corners of the existence domain in Fig. 11 now

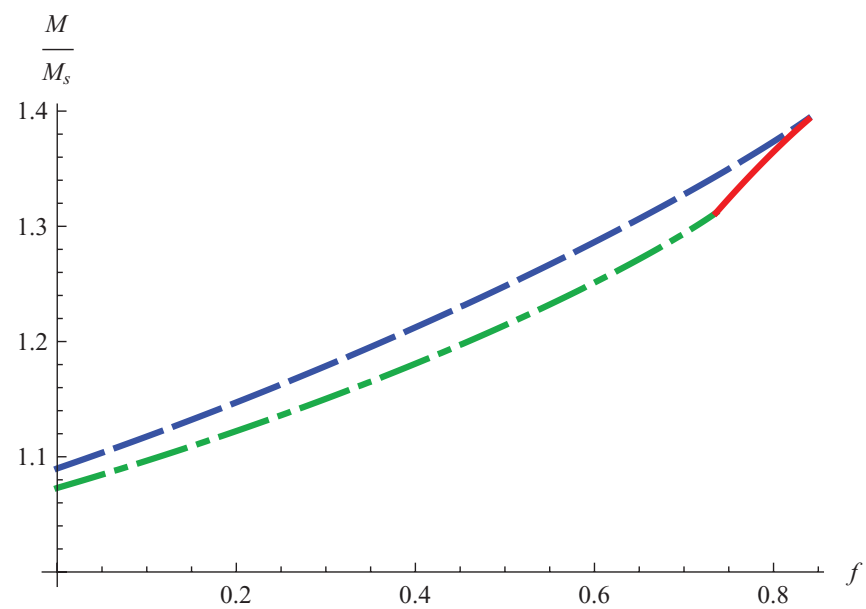

FIG. 11. (Color online) Region where negative supersolitons can be found, for $\beta=0.5$, above the green dotted-dashed curve (occurrence of negative double layers) or the solid red curve ( $A B$ coalescence) and below the blue dashed curve ( $B C$ coalescence). There are no supersolitons at the acoustic speed. 


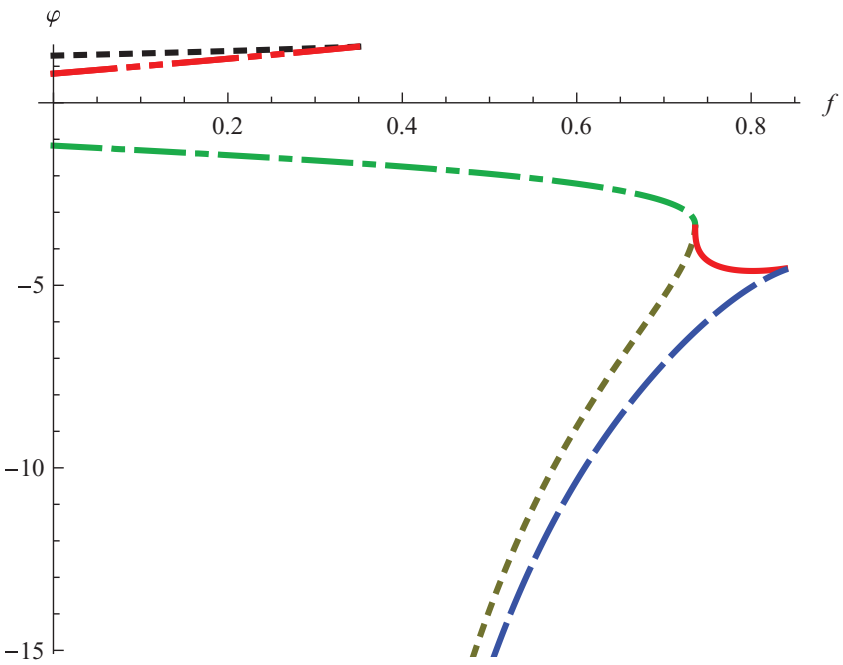

FIG. 12. (Color online) Existence ranges for positive and negative solitons and supersolitons, for $\beta=0.5$, in terms of the amplitudes, with the same curve coding as in Fig. 3. In this case, too, only a part of the blue dashed and light gray lower dotted curves are illustrated, as for decreasing $f$ the limiting amplitudes increase very rapidly.

occur for $f=0$ (though not physical), $f=0.736$ (triple root at the end of the double-layer range), and $f=0.839$ (coalescence of all three local extrema).

However, when drawing in Fig. 12 the equivalent of Fig. 3 for the amplitude ranges, with the same curve coding, the supersoliton amplitudes are shown to be rather strong. Only for relatively large $f$ (relative charge density), which signals an appreciable electron depletion as a prerequisite, do they have amplitudes of more usual magnitude.

Once the existence diagrams are obtained, it is straightforward to draw some Sagdeev pseudopotentials and corresponding hodographs, supersoliton electrostatic potentials, and electric field profiles. They qualitatively resemble those described in the previous subsection, and those of our earlier paper [13], in a striking way, hence, a single example will suffice (for $f=0.736$ ), where the double-layer range ends in a triple root, as $f$ is increased. This is the analog for $\beta=0.5$ of Fig. 7 and the results are similar. One sees from Fig. 12 that this case lies beyond the range of positive solitons, and, thus, only negative potential solitons are found.

This pseudopotential is illustrated in the upper panel of Fig. 13 (green dashed curve), together with pseudopotentials having a normal soliton (blue dotted curve) or a supersoliton (red solid curve). In the lower panel the corresponding hodographs are given, plotting $d \varphi / d x$ as functions of $\varphi$. The pseudopotential plotted in Fig. 13 (solid curve) yields a supersoliton profile and associated electric field, as shown in Fig. 14. It has to be remarked again that there are always negative solitons, but beyond the double layers their amplitudes jump to surprisingly large values, as there is no limit for negative potentials in the model. That is the case because the negative charges are carried by massless electrons and infinitely massive dust grains, unlike the case reported in Ref. [10], where the positive supersolitons beyond the

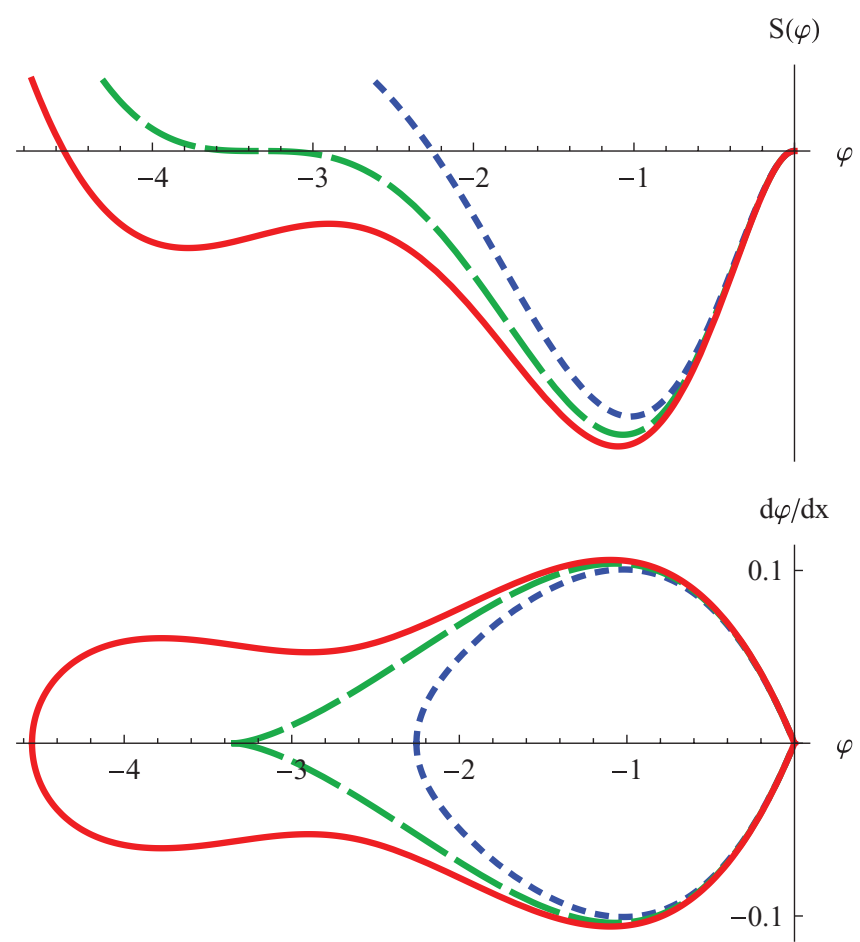

FIG. 13. (Color online) Upper panel: Pseudopotentials with a triple root (green dashed curve, $M / M_{s}=1.312$ ), with a standard soliton (blue dotted curve, $M / M_{s}=1.305$ ), and with a supersoliton (red solid curve, $M / M_{s}=1.316$ ) solution for $\beta=0.5$ and $f=0.736$. There are no positive solitons here. Lower panel: Corresponding hodographs, plotting $d \varphi / d x$ as functions of $\varphi$.

double-layers experience a cutoff due to infinite compression of the finite mass ions.

\section{Model limitations on Mach numbers and amplitudes}

Having discussed some specific cases for $\beta=0.3$ and 0.5 , we need to address the limitations of our model when confronted with the theoretical possibility of obtaining very large soliton and supersoliton amplitudes. Part of this is reminiscent of earlier work [17], but we focus here on the neglect of dust dynamics and/or of electron inertia.

To begin with the dust dynamics, had we included it from the beginning and treated the charged dust as a (very) heavy negative-ion species, we would have found, by analogy with the cold positive-ion density, that

$$
n_{d}=\frac{1}{\sqrt{1+\frac{2 \varphi}{\mu M^{2}}}} .
$$
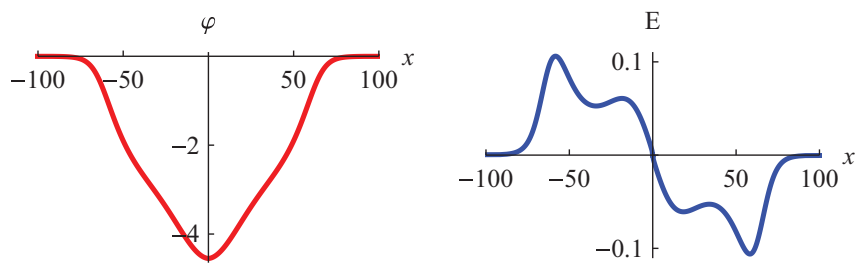

FIG. 14. (Color online) Soliton (left) and electric field (right) profile for the pseudopotential shown by the red solid curve in Fig. 13. 
Here $\mu=z_{i} m_{d} / z_{d} m_{i}$ is the ratio of the dust-to-ion mass-percharge ratios, for charged dust usually a very large number. In turn, this means that the Sagdeev pseudopotential in (5) becomes

$$
\begin{aligned}
S(\varphi, M)= & (1-f)\left[1+3 \beta-\left(1+3 \beta-3 \beta \varphi+\beta \varphi^{2}\right) \exp (\varphi)\right] \\
& +f \mu M^{2}\left(1-\sqrt{1+\frac{2 \varphi}{\mu M^{2}}}\right) \\
& +M^{2}\left(1-\sqrt{1-\frac{2 \varphi}{M^{2}}}\right)
\end{aligned}
$$

That is, the dust contribution, $-f \varphi$, is replaced in (5) by

$$
f \mu M^{2}\left(1-\sqrt{1+\frac{2 \varphi}{\mu M^{2}}}\right) .
$$

This introduces a limit $\varphi_{\ell d}=-\mu M^{2} / 2$ on the negative $\varphi$ range, with magnitude $\left|\varphi_{\ell d}\right| \gg \varphi_{\ell i}$. For large but finite $\mu$, and, thus, very large negative solitary waves, $\varphi \rightarrow \varphi_{\ell d}$, the electron term $\left(\propto \exp \left[\varphi_{\ell d}\right]\right)$ is negligible. Thus, the sign of $S(\varphi, M)$ involves a subtle balance between the two remaining potentially large terms for small dust charge density $f$, namely

$$
M^{2}(1-\sqrt{1+\mu})+f \mu M^{2} \simeq M^{2} \sqrt{\mu}(f \sqrt{\mu}-1) .
$$

The limit $f=0$ represents a two-species (electron-ion) plasma, for which the factor $\mu$ plays no role, and in that case the dust term in $S(\varphi, M)$ then falls away completely. But in the neighborhood of $f=0, S(\varphi, M)>0$ at $\varphi_{\ell d}$, i.e., a root exists before the cutoff is reached, only holds if $f>1 / \sqrt{\mu}$.

For typical dust masses and charges $\mu$ is very large, implying that $f>1 / \sqrt{\mu}$ only excludes a very narrow range near $f=0$. However, such small $f$ mean that there is hardly any dust, which invalidates the DIA model. Seemingly, this constraint does not involve $M$, and, hence, if $M$ can be increased at will, both $\varphi_{\ell i}$ and $\varphi_{\ell d}$ could become very large, unless other physical restrictions appear.

Similar conclusions can be reached for a model in which the electrons are governed by $\kappa$ distributions [25,27], which include as a special case, for $\kappa \rightarrow \infty$, the Boltzmann distribution used in Ref. [4]. In particular, a detailed discussion [24] shows that neglecting dust dynamics hardly affects the description of the positive solitons and that there is, for large dust mass, only a tiny range at small $f$ where the limit due to $\varphi_{\ell d}$ plays a role. Specifically, for a model that includes analogous noninertial electrons (Boltzmann $/ \kappa$ distributed), Ref. [24], too, yields very large amplitude non-KdV-like negative solitons, even though they have included warm ions and dust grains of finite mass. Nonetheless, the question must be asked as to whether these large amplitudes are indeed physically plausible or whether the assumption of massless electrons or some other artifact of the model has given rise to them.

Electron inertia comes into play in a totally different way. The electrons have been assumed hot enough so their inertia can be neglected, and this requires that $V \ll c_{t e}$, with $c_{t e}=$ $\sqrt{T_{e} / m_{e}}$ defining the electron thermal velocity, up to factors of $(1-\beta)$, of order unity. Given our normalization, this is equivalent to requiring that $M \ll \sqrt{m_{i} / z_{i} m_{e}}$. While this limit is large enough not to bother us when focusing on some of the supersoliton solutions, it is not infinite and $M$ therefore should be restricted to "reasonable" values. This is, however, not easy to quantify. Such considerations are not limited to this problem alone but might be encountered for various other plasma compositions, which explicitly or tacitly assume the hot species to be completely inertialess.

\section{SUMMARY}

Based on the recently introduced concept of supersolitons, we have revisited the problem of large-amplitude DIA waves in a plasma consisting of cold fluid protons and Cairns nonthermal electrons in the presence of a neutralizing negative dust background. This has been based on a Sagdeev pseudopotential method, because supersolitons always have finite amplitudes and cannot be treated by reductive perturbation techniques.

The picture which emerges from this model is that supersolitons are usually wedged between ranges of ordinary solitons and have negative polarity. If double layers can occur, supersolitons have Mach numbers that are larger than those of the double-layer pseudopotential but amplitudes which jump discontinuously from the double-layer amplitude to beyond the third (nonaccessible) root of the double-layer pseudopotential. Besides supersolitons, one also finds the usual solitons, some of which can have positive polarity, and there are usually appreciable ranges of coexistence.

We have illustrated these findings for two specific values of the nonthermality parameter, and have also included a subsection on the model limitations, because the neglect of dust dynamics and electron inertia eliminates all upper bounds on Mach number and amplitudes for the negative-polarity solitons. Thus, unphysically large structures might be found which ultimately will invalidate the simplifying hypotheses.

According to our findings, the supersoliton range is always bounded from below or above, in this sense. Indeed, one of the supersoliton characteristics is that the corresponding pseudopotential must have two contiguous subwells in the electrostatic potential region between the (sole) root and the undisturbed conditions at zero electrostatic potential. As the Mach number increases these two subwells are merged, so there are no longer supersolitons. It can be shown numerically that there is a lower limit in $\beta$ below which supersolitons cannot be found, in particular, not for Maxwellian electrons in this DIA model.

At this stage it is not possible to give a clear indication of all possible classes of plasma models that could support supersolitons. A minimum requirement would be that there are a sufficient number of species to generate the complicated Sagdeev pseudopotential that represents a supersoliton. From our experience of related calculations we believe that twospecies models do not have sufficient complexity to give rise to such curves, whereas three-species models certainly allow for them. In Ref. [8] the authors speculate that bipolar and tripolar solitons can be explained in terms of BernsteinGreen-Kruskal (BGK) modes in ordinary two-component plasmas. They do not appear to comment explicitly on the single "modified" bipolar structure which appears in their Fig. 1(b). It is suggested that supersolitons might offer an alternative explanation to BGK modes for such solitary waves. 
What other constraints are required is still unclear, but certainly supersolitons have now been found in three models of diverse physical makeup. Here we considered a plasma with an inertial and an immobile species of opposite polarity, together with a Cairns nonthermal species, in Ref. [13] the two mobile inertial species also had opposite charge, and again a nonthermal electron component was included, while in Ref. [10] the plasma involved two Boltzmann distributions of the same polarity and a single mobile species. Thus, the presence of a so-called nonthermal species is certainly not a prerequisite.

\section{ACKNOWLEDGMENTS}

M.A.H. thanks the South African National Research Foundation for support. I.K. warmly acknowledges support from the UK Engineering and Physical Sciences Research Council (EPSRC) via Grant No. EP/I031766/1.
[1] F. Verheest, Waves in Dusty Space Plasmas (Kluwer Academic Publishers, Dordrecht, 2000).

[2] P. K. Shukla and A. A. Mamun, Introduction to Dusty Plasma Physics (IOP Press, London, 2002).

[3] P. K. Shukla and V. P. Silin, Phys. Scr. 45, 508 (1992).

[4] R. Bharuthram and P. K. Shukla, Planet. Space Sci. 40, 973 (1992).

[5] R. Z. Sagdeev, Reviews of Plasma Physics, edited by M. A. Leontovich (Consultants Bureau, New York, 1966), Vol. 4, p. 23.

[6] A. E. Dubinov and D. Yu. Kolotkov, IEEE Trans. Plasma Sci. 40, 1429 (2012).

[7] A. E. Dubinov and D. Yu. Kolotkov, High Energy Chemistry 46, 349 (2012).

[8] J. S. Pickett, L.-J. Chen, S. W. Kahler, O. Santolík, D. A. Gurnett, B. T. Tsurutani, and A. Balogh, Ann. Geophys. 22, 2515 (2004).

[9] F. Verheest, Phys. Plasmas 16, 013704 (2009).

[10] T. K. Baluku, M. A. Hellberg, and F. Verheest, Europhys. Lett. 91, 15001 (2010).

[11] F. Verheest, Phys. Plasmas 18, 083701 (2011).

[12] A. Das, A. Bandyopadhyay, and K. P. Das, J. Plasma Phys. 78, 149 (2012).

[13] F. Verheest, M. A. Hellberg, and I. Kourakis, Phys. Plasmas 20, 012302 (2013).

[14] R. A. Cairns, A. A. Mamun, R. Bingham, R. Boström, R. O. Dendy, C. M. C. Nairn, and P. K. Shukla, Geophys. Res. Lett. 22, 2709 (1995).

[15] J. F. McKenzie, Phys. Plasmas 9, 800 (2002).

[16] F. Verheest, T. Cattaert, G. S. Lakhina, and S. V. Singh, J. Plasma Phys. 70, 237 (2004).

[17] F. Verheest, T. Cattaert, and M. A. Hellberg, Phys. Plasmas 12, 082308 (2005).

[18] F. Sayed, M. M. Haider, A. A. Mamun, P. K. Shukla, B. Eliasson, and N. Adhikary, Phys. Plasmas 15, 063701 (2008).

[19] M. A. Hellberg, F. Verheest and T. Cattaert, J. Phys. A: Math. Gen. Phys. 39, 3137 (2006).
[20] A. A. Mamun, N. Jahan, and P. K. Shukla, J. Plasma Phys. 75, 413 (2009).

[21] A. A. Mamun, P. K. Shukla, and B. Eliasson, Phys. Rev. E 80, 046406 (2009).

[22] M. Bacha, M. Tribeche, and P. K. Shukla, Phys. Rev. E 85, 056413 (2012).

[23] S. K. El-Labany, W. F. El-Taibany, and M. M. El-Fayoumy, Astrophys. Space Sci. 341, 527 (2012).

[24] T. K. Baluku, M. A. Hellberg, I. Kourakis, and N. S. Saini, Phys. Plasmas 17, 053702 (2010).

[25] V. M. Vasyliunas, J. Geophys. Res. 73, 2839 (1968).

[26] T. K. Baluku and M. A. Hellberg, Phys. Plasmas 15, 123705 (2008).

[27] M. A. Hellberg, R. L. Mace, T. K. Baluku, I. Kourakis, and N. S. Saini, Phys. Plasmas 16, 094701 (2009).

[28] F. Verheest and S. R. Pillay, Phys. Plasmas 15, 013703 (2008).

[29] F. Verheest, M. A. Hellberg, and T. K. Baluku, Phys. Plasmas 19, 032305 (2012).

[30] F. Verheest, Phys. Plasmas 17, 062302 (2010).

[31] S. Baboolal, R. Bharuthram, and M. A. Hellberg, J. Plasma Phys. 44, 1 (1990).

[32] C. A. Mendoza-Briceño, S. M. Russell, and A. A. Mamun, Planet. Space Sci. 48, 599 (2000).

[33] F. Verheest and M. A. Hellberg, Phys. Plasmas 17, 102312 (2010).

[34] F. Verheest, M. A. Hellberg, N. S. Saini, and I. Kourakis, Phys. Plasmas 18, 042309 (2011).

[35] A. A. Mamun, R. A. Cairns, and P. K. Shukla, Phys. Plasmas 3, 2610 (1996).

[36] A. A. Mamun, Phys. Rev. E 55, 1852 (1997).

[37] A. A. Mamun, S. M. Russell, C. A. Mendoza-Briceño, M. N. Alam, T. K. Datta, and A. K. Das, Planet. Space Sci. 48, 163 (2000).

[38] M. Asaduzzaman and A. A. Mamun, Phys. Rev. E 86, 016409 (2012)

[39] W. Masood and A. Ahmad, Astrophys. Space Sci. 340, 367 (2012). 